\title{
Treatment of MGMT promoter unmethylated glioblastoma with PD-1 inhibitor combined with anti-angiogenesis and epidermal growth factor receptor tyrosine kinase inhibitor: a case report
}

\author{
Zheng Wang", Fangfang Dü, Yi Ren, Wei Jiang \\ Department of Radiation Oncology, Tianjin Huanhu Hospital, Tianjin, China \\ \#These authors contributed equally to this work. \\ Correspondence to: Wei Jiang. Department of Radiation Oncology, Tianjin Huanhu Hospital, 6 Jizhao Road, Jinnan, Tianjin 300060, China. \\ Email: weijiangthh@163.com.
}

\begin{abstract}
Glioblastoma (GBM) is the most common primary central nervous system (CNS) malignancy in adults and is associated with poor prognosis, especially even worse in those with unmethylated MGMT promoter. Currently, maximal safe resection combined with temozolomide (TMZ) concurrent chemoradiotherapy and TMZ adjuvant chemotherapy has been considered the standard treatment for newly diagnosed GBM. The efficacy of drugs other than TMZ is currently undefined. With increasing understanding of the biological characteristics of GBM, more and more studies are being conducted on drug targets, such as specific signaling pathways and microenvironment. Herein, we report the case of a GBM patient with unmethylated MGMT promoter who was intolerant to TMZ, and underwent treatment with the combination of carelizumab, anlotinib, and oxitinib during radiotherapy according to results of wholeexome sequencing (WES) and the patient's condition. The progression-free survival (PFS) and overall survival (OS) for this case were respectively nearly 11 and 18 months, significantly exceeding the historical data and the tolerance of the treatment for this case without sever adverse effects was favorable. Our case provides clinical evidence supporting the efficacy of the above three drugs and radiotherapy, which may translate into novel individualized treatment strategies for GBM patients who are intolerant to TMZ.
\end{abstract}

Keywords: Oxitinib; carelizumab; anlotinib; glioblastoma (GBM); case report

Submitted Aug 10, 2021. Accepted for publication Oct 02, 2021.

doi: $10.21037 / \mathrm{atm}-21-4625$

View this article at: https://dx.doi.org/10.21037/atm-21-4625

\section{Introduction}

Glioblastoma (GBM) is the most common primary malignant tumor of the adult central nervous system (CNS), and has a poor prognosis. The current standard treatment involves a combination of temozolomide (TMZ) concurrent chemoradiotherapy and TMZ adjuvant chemotherapy based on maximal safe resection, with a median overall survival (OS) of only 14-16 months and a 5-year survival rate of no more than $10 \%$ (1). MGMT promoter methylation is a good prognostic factor for survival, thus the median OS of GBM with MGMT promoter unmethylated receiving standard treatment is only 11-14 months (2).

With advancements in the basic research, such as in the field of molecular biology, it is now understood that epidermal growth factor receptor (EGFR)-related signaling pathways are closely correlated with glioma grade and prognosis. Relevant studies have shown that most of the primary GBMs involve EGFR gene amplification, rearrangement, and mutation. This in turn led to the activation of downstream signaling pathways, providing a new therapeutic target (3). The CNS tumor classification according to the World Health Organization (WHO; 2016) includes molecular features based on the histology for the first time, dividing GBM into isocitrate dehydrogenase (IDH) wild-type and IDH mutant type. According to the research report of the Consortium to Inform Molecular 

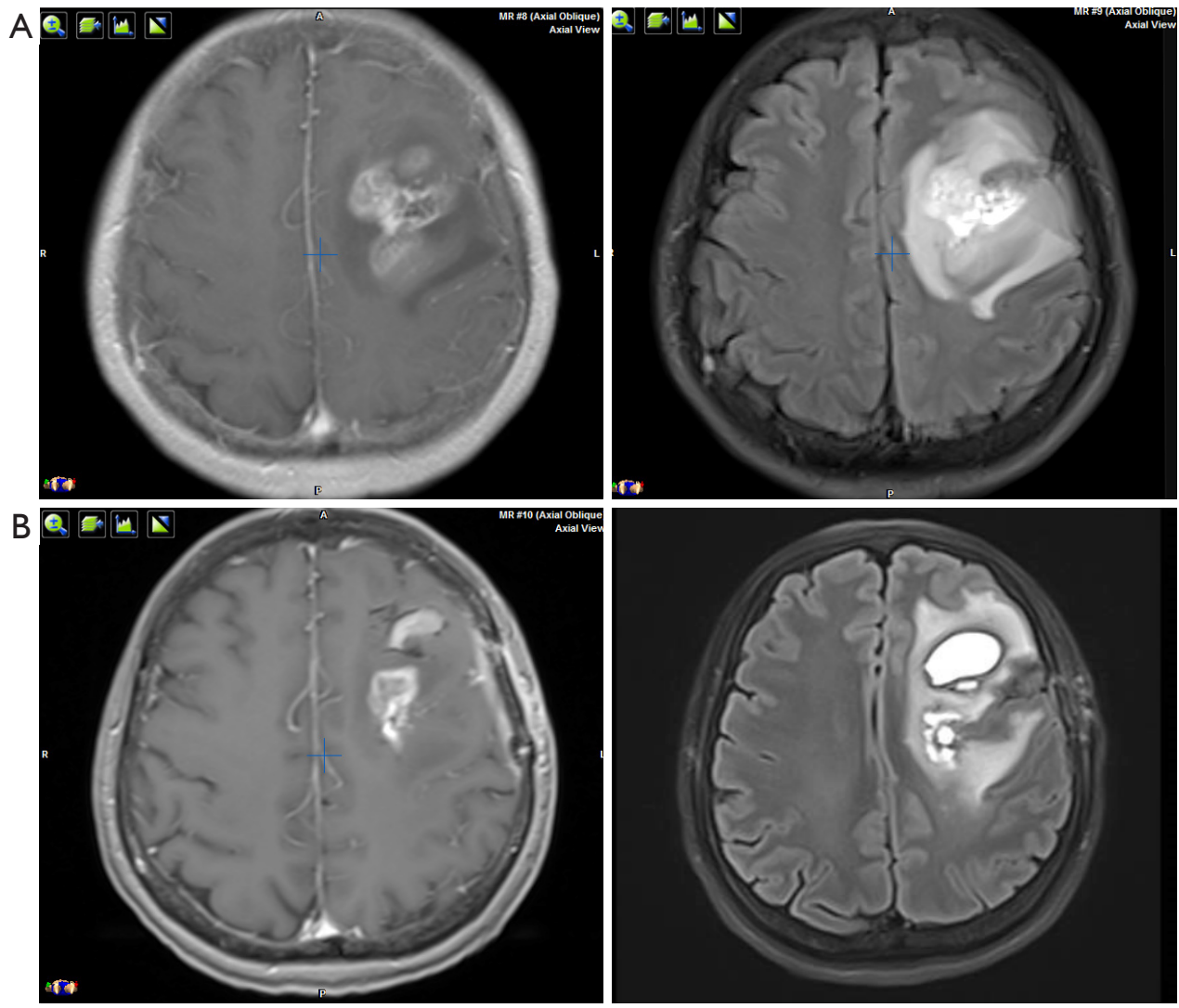

Figure 1 MRI image from this case. Right: FLAIR sequence, left: T1 enhancement sequence. (A) Pre-operative MRI findings; (B) preradiotherapy MRI findings. MRI, magnetic resonance imaging.

and Practical Approaches to CNS Tumor Taxonomy (a non-WHO official organization), IDH wild-type diffuse astrocytoma or anaplastic astrocytoma that lack histological features of GBM might have any of the following molecular genetic characteristics: EGFR amplification; chromosome 7 acquisition/chromosome 10 deletion; and telomerase reverse transcriptase (TERT) promoter mutation, and all be diagnosed as diffuse astrocyte glioma, IDH wildtype, with GBM molecular pathology characteristics, and WHO IV. With increased understanding of the biological characteristics of GBM, there have been an increasing number of drug studies that target specific signaling pathways and microenvironment, such as targeted drugs, antivascular drugs and immunotherapy. however, these drugs offer no obvious improvement for GBM by themselves alone.

We herein report a GBM case who was intolerant to TMZ, and so underwent treatment with the combination of carelizumab, anlotinib, and oxitinib during radiotherapy, achieving long-term progression-free survival (PFS) and
OS. We present the following article in accordance with the CARE reporting checklist (available at https://dx.doi. org/10.21037/atm-21-4625).

\section{Case presentation}

A 56-year-old female was admitted to the hospital due to "motor aphasia for more than a week". She did not have any specific history of medication or malignant tumor. Cranial computed tomography (CT, plain scan) taken in other hospital showed a space-occupying lesion in the left frontal lobe. Preoperative cranial magnetic resonance imaging (MRI) showed a space-occupying lesion in the left frontal lobe, with a high and slightly high signal intensity on T2weighted image (T2WI) and a slightly low and low signal intensity on T1WI. After enhanced scanning, the lesion in the left frontal lobe showed obvious enhancement with nonuniform signals, and the lesion was about $3.4 \mathrm{~cm} \times 4.2 \mathrm{~cm} \times$ $3.0 \mathrm{~cm}$ (Figure 1A). The patient then underwent craniotomy under general anesthesia on 12 July 2019. Postoperative 
pathology revealed GBM, IDH wild-type (WHO grade IV). The results of whole-exome sequencing (WES) (GenomiCare Biotechnology Co., Ltd., Shanghai, China) are shown in Tables 1,2. At 3 weeks after the operation (6 August 2019), oral administration of Stupp's regimen (TMZ, $75 \mathrm{mg} / \mathrm{m}^{2}$ d) orally with concurrent radiotherapy) was commenced. Pre-radiotherapy MRI (plain scan + enhanced) examination showed irregular abnormal signals on the left frontal parietal region, and patchy heterogeneous hyperintense signal shadow after enhanced scan, with surrounding edema (Figure $1 \mathrm{~B}$ ). Next, conventional fractionation $(60 \mathrm{~Gy} / 30 \mathrm{~F})$ intensity-modulated radiotherapy was used. The radiation dose distribution is shown in Figure 2. In addition to treatment-related grade III agranulocytosis and grade II thrombocytopenia, the patient also had significant gastrointestinal adverse reactions, such as fatigue and decreased appetite, during concurrent chemoradiotherapy. After suspending the TMZ chemotherapy and symptomatic treatment, the symptoms improved and the bone marrow function was recovered. Subsequently, the patient refused to take TMZ again due to poor tolerance to chemotherapy, and so they was given carelizumab (200 mg Q21d) and oxitinib (80 $\mathrm{mg}$ Qd) during radiotherapy. During this period, the patient had good overall tolerance and completed radiotherapy smoothly. At 3 weeks after radiation, the

Table 1 Summary of tumor mutation spectrum

\begin{tabular}{lc}
\hline Parameters & Value \\
\hline Tumor mutational burden (TMB) & 128 \\
\% Microsatellite Instability Analysis (MSI) & 0.21 \\
\% Copy-number Variation (CNV) & 3.10 \\
\hline
\end{tabular}

patient had symptoms of intermittent headaches, which are gradually aggravated. Head MRI (plain scan + enhanced) (23 October 2019) showed that the peritumoral edema was larger than that of the anterior area when compared to the previous images. Perfusion weighted MRI showed hypoperfusion changes in the left frontal lesion area (Figure $3 A$ ). The possibility of pseudoprogression was considered by incorporating the past treatment history through multidisciplinary consultation. Anlotinib $(8 \mathrm{mg}$ Qd) in combination with carelizumab and oxitinib was used for anti-angiogenic therapy due to the symptoms of increased intracranial pressure. Head MRI reexamination 2 months after radiotherapy (23 November 2019) showed a narrowed edema area and near disappearance of the original abnormal enhancement signal in the lesion area (Figure 3B). Subsequently, the original regimen of carelizumab, oxitinib, and anlotinib was continued, and the patient underwent

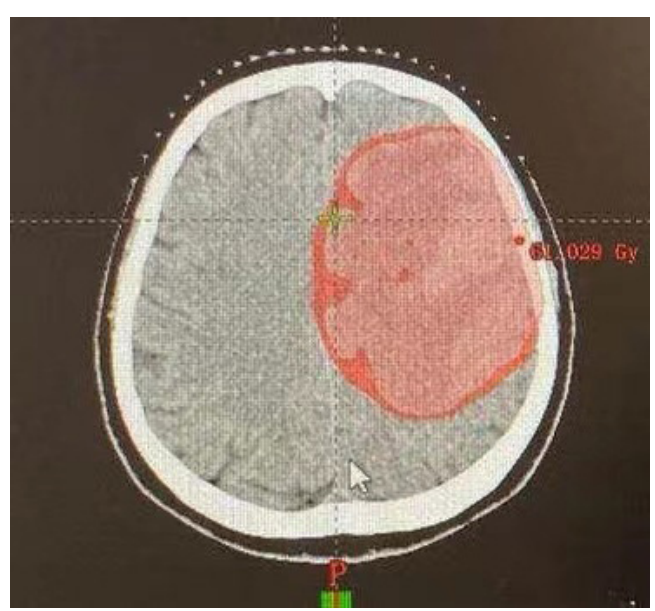

Figure 2 Dose distribution.

Table 2 List of key tumor drivers

\begin{tabular}{|c|c|c|c|c|c|}
\hline Mutant gene & $\begin{array}{l}\text { Signaling pathway/molecular } \\
\text { mechanism }\end{array}$ & $\begin{array}{c}\text { Mutation site/mutation } \\
\text { state }\end{array}$ & $\begin{array}{c}\text { Frequency/copy } \\
\text { number }\end{array}$ & $\begin{array}{c}\text { Matching of } \\
\text { marketed drugs }\end{array}$ & $\begin{array}{l}\text { Matching of } \\
\text { clinical trial }\end{array}$ \\
\hline$C D K N 2 A$ & Cell cycle & Deletion & 0.8 & Yes & Yes \\
\hline EGFR & ErbB family & p.A289V & $86.50 \%$ & Yes & Yes \\
\hline PTEN & PI3K/Akt/mTOR & Deletion & 1.04 & Yes & Yes \\
\hline TP53 & P53 & p.E180D & $38.24 \%$ & No & Yes \\
\hline
\end{tabular}


A
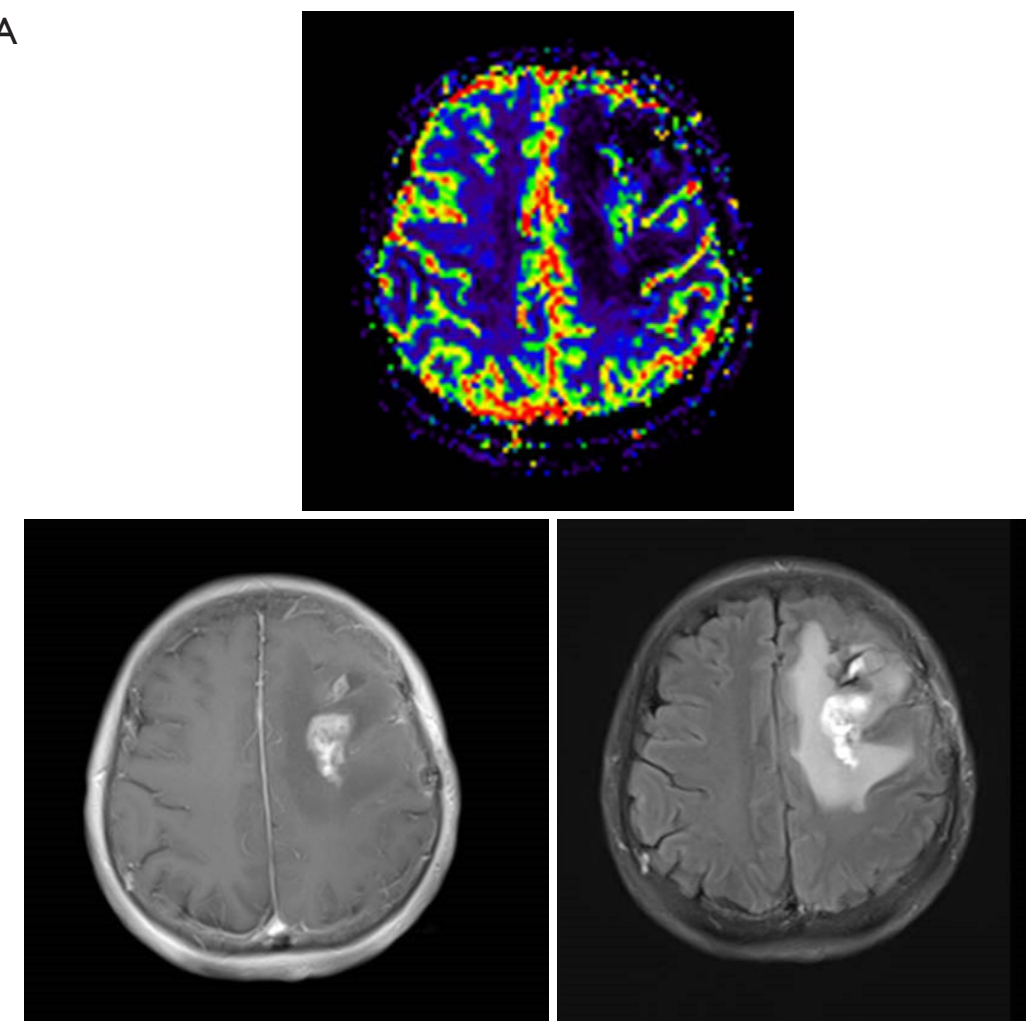

B
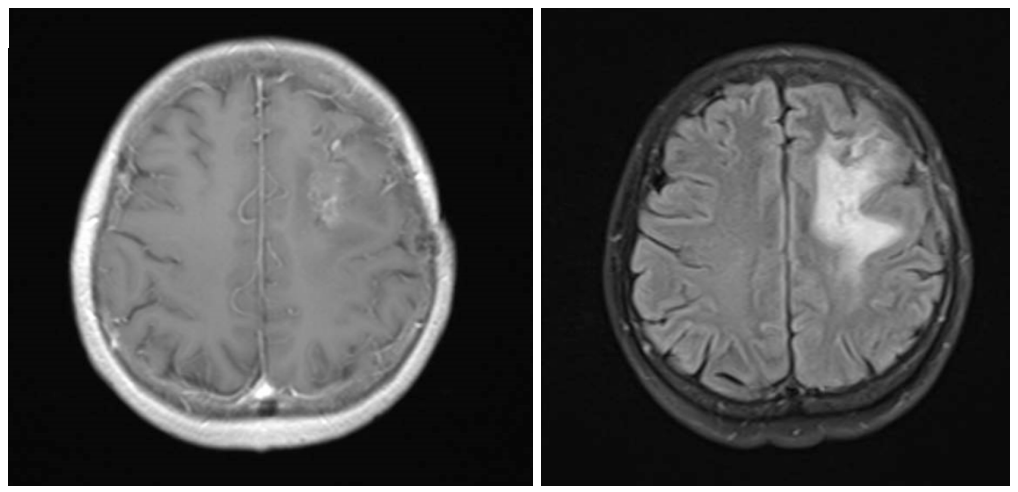

C
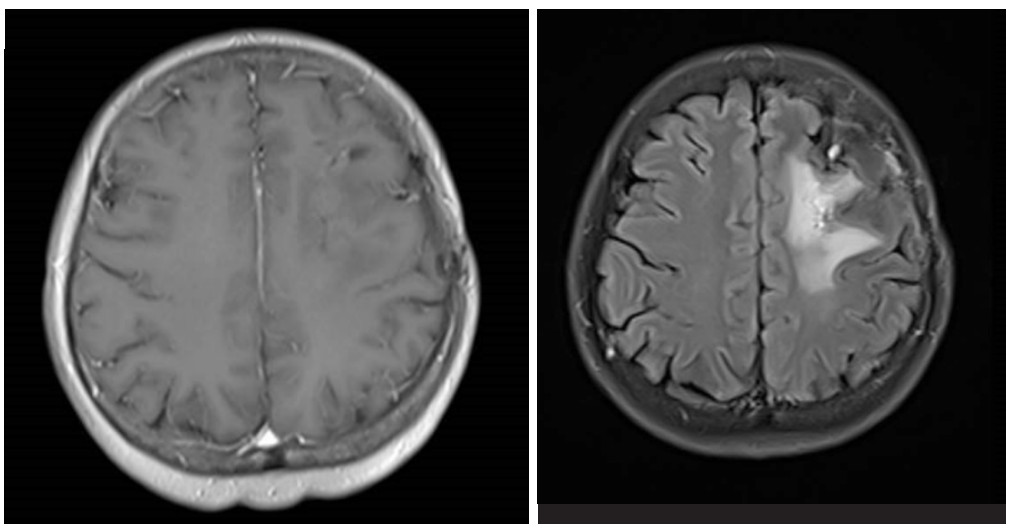

Figure 3 MRI image from this case. Right: FLAIR sequence, left: T1 enhancement sequence. (A) Plain scan + enhanced MRI and perfusion imaging 1 month after radiotherapy; (B) MRI findings of carelizumab and oxitinib based on the addition of anlotinib after 1 month; (C) recent reexamination of MRI findings. MRI, magnetic resonance imaging. 


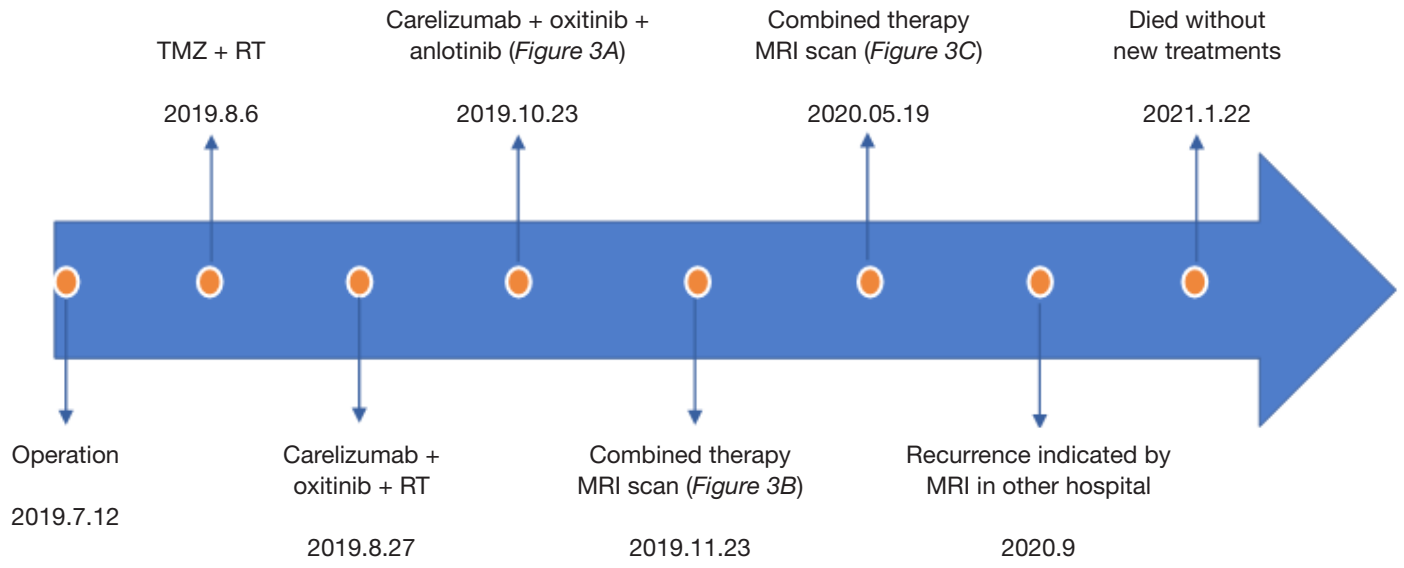

Figure 4 Timeline and duration of each treatment. TMZ, temozolomide; RT, radiotherapy; MRI, magnetic resonance imaging.

regular examinations by MRI. About 11 months after the operation, the quality of life and the tolerance to targeted therapy and immunotherapy of the patient remained good, and no tumor progression was observed in MRI reexamination performed on 19 May 2020 (Figure 3C). About 4 months after the last MRI reexamination, the patient had symptoms of intermittent headaches again. MRI in other hospital indicated progression, but we didn't get the image. The patient refused any examination and any other new treatments except for mannitol decreasing intracranial pressure and died on 22 January 2021. The timeline is shown in Figure 4.

\section{Ethical statement}

All procedures performed in studies involving human participants were in accordance with the ethical standards of the institutional and/or national research committee(s) and with the Helsinki Declaration (as revised in 2013). Written informed consent was provided by the patient's families. The patient's families have given the consent for the publication of this case. A copy of the written consent is available for review by the editorial office of this journal.

\section{Discussion}

Although Stupp's regimen has been shown to significantly improve the PFS and OS in patients with GBM, the same results in those with unmethylated MGMT promoter was not detected (2). In order to increase the TMZ sensitivity due to unmethylated MGMT promoter, TMZ with different doses were suggested. There was no significant advancement in
OS and PFS by prescribing a DD TMZ in newly diagnosed GBM, regardless of MGM methylation (4). Several recent studies have attempted to improve the therapeutic efficacy of GBM patients with poor prognosis through other treatments, such as targeted therapy, immunotherapy, and tumor-treating fields (TTFields). In this report, poor treatment tolerance (grade III myelosuppression and grade IV gastrointestinal reaction) during standard Stupp's regimen treatment postoperatively was observed. Combined with the results of WES, the immune checkpoint inhibitor carelizumab, oxitinib which is the new EGFR tyrosine kinase inhibitor (TKI), and the multi-target antivascular anlotinib, were used for individualized combination therapy as the replacement of chemotherapy.

The programmed cell death (PD) pathway is one of the mechanisms by which tumors, including GBM, negatively regulate $\mathrm{T}$ cell viability. Iwai et al. reported the importance of PD-1/PD-L1 in tumor immunity for the first time. Blocking PD-1/PD-L1 pathway by antibodies can significantly restore the anti-tumor immune response and promote lymphocytes to attack tumor cells (5). Previous studies have suggested that brain metastases secondary to malignant melanoma and non-small cell lung cancer have achieved encouraging results with PD1 monoclonal antibody therapy (6). Although the safety with regard to the application of PD-1/PD-L1 antibody in GBM has been currently confirmed in relevant trials (7), the curative effect has remained unsatisfactory when compared with body tumors (8). A related clinical trial for recurrent GBM showed that the single-drug response rate of PD-1 inhibitor is only $8 \%$ (9). The possible reason for this is that it is closely related to the unique physiological structure 
of the nervous system, the immune mechanism, and the immune environment of GBM. To date, studies on immune checkpoint inhibitors in the treatment of malignant glioma have focused on combination therapy, including surgery, radiotherapy, chemotherapy, and targeted therapy. The WES result showed a high tumor mutation burden (TMB), which is an immunotherapy benefit factor, thus adopting PD-1 inhibitor.

Radiotherapy is an important component of the standard treatment of GBM. Recent studies have confirmed that radiotherapy can activate many molecular and transcriptional pathways, which in turn regulates the immune response and alters the immune phenotype of the tumor cells (10). These findings open a new field of research for radiotherapy of CNS, namely radiotherapy combined with immunotherapy. Relevant preclinical studies have confirmed the synergistic effects of PD1 inhibitor combined with radiotherapy (11). Although the PD-1 inhibitor combined with radiotherapy for GBM has achieved no positive results in recent clinical trials, the screening of the population benefiting from immunotherapy might be a key factor (Checkmate 498). Compared with traditional chemotherapy, better treatmentrelated tolerance and less adverse reactions were reported with immunotherapy combined with radiotherapy (12). Chemotherapy was terminated due to treatment toxicities during Stupp's regimen treatment in our patient. Due to higher TMB of the patient and the synergistic effects of radiotherapy and immunotherapy, radiotherapy combined with carelizumab was delivered.

The highly vascularized features of GBM have shown a close association with poor prognosis. Therefore, antiangiogenic therapy has become a research hotspot in the treatment of GBM. There are many angiogenic modes in GBM, including sprouting growth, mimicry of angiogenesis, chimeric vessels, and vascular intussusception. The more common angiogenic factors include vascular endothelial growth factor (VEGF), fibroblast growth factor (FGF), platelet-derived growth factor (PDGF), and so on. The higher the malignancy degree of glioma, the higher the expression of VEGF. Current studies have shown that VEGF not only promotes angiogenesis, but is also closely related to regulation of the tumor immune microenvironment (13). Relevant mechanisms such as blocking $\mathrm{T}$ cell mobilization by inhibiting lymphocyte adhesion to activate endothelial cells and interference with Fas ligand might affect lymphocyte infiltration into the tumors $(14,15)$. This in turn induces proliferation of suppressive immune cell subtypes such as regulatory
T cells (Tregs) and myeloid-derived suppressor cells (MDSCs), inhibiting the maturation of dendritic cells (DC) and the development of T cells derived from hematopoietic progenitor cells (16). Therefore, therapeutic strategies targeting VEGF or its receptors can play a role in stimulating the immune response, thus increasing the efficacy of immunotherapy. Increased therapeutic efficacy of antiangiogenic drugs combined with immunotherapy has also been supported by preclinical data obtained from several tumor models (17), and bevacizumab improved CD4+, CD8+ T cells and CD20+ B cells in the peripheral blood (18). In addition, pseudoprogression occurred after radiotherapy. The humanized anti-VEGF monoclonal antibody bevacizumab has been approved by the US Food and Drug Administration for the treatment of recurrent GBM in 2009, showing a significant effect of it in the treatment of radiation necrosis. Previous studies have confirmed that radiation necrosis and pseudoprogression share similar pathological features, and so blockage of VEGF pathway can be used for the treatment of pseudoprogression. Other antiangiogenic drugs are also used for the treatment of GBM. As a multi-target antivascular drug, axitinib has been shown to effectively inhibit angiogenesis-related targets such as VEGF receptor (VEGFR)1-3, PDGF receptor (PDGFR), and c-Kit. A phase II study showed that the objective response rate of axitinib in the treatment of recurrent GBM was $28 \%$, the 6 -month disease-free progression rate was $34 \%$, and the average OS was 29 weeks, which was higher than that of the control group and so is considered to be safe (19). Due to pseudoprogression of the patient with cranial hypertension symptoms and the synergistic treatment effects with immunotherapy, our patient was treated with anlotinib (made in China), which is highly similar to the target of axitinib treatment. The patient tolerated this treatment well, experiencing only oral erythema and pain, which were improved with symptomatic treatment. Similarly, antivascular drug plus immunotherapy show a satisfactory result in recurrent GBM (20).

The EGFR gene is one of the important pathogenic genes of primary GBM. Nearly half of the patients with GBM are accompanied with EGFR overexpression and EGFR gene amplification. Our patient with IDH wild-type GBM had both EGFR amplification and EGFR (p.A289V) mutation. Activation of EGFR activates downstream signaling pathways such as Ras-Raf-MAPK and PI3K-AKT, thereby increasing tumor proliferation, survival, angiogenesis, and migration. In addition, the EGFR signaling pathway promotes the escape 
of tumor cells from host anti-tumor immunity through the following aspects: (I) upregulation of PD-L1 expression is done by activating ERK, AKT-mTOR and STAT3; (II) inhibition of activity of CTLs by increasing lactate excretion; (III) increasing Treg cells by activating GSK-3b/Foxp3; and (IV) reduction of $\mathrm{MHCI}$ and $\mathrm{MHCII}$ expression by inducing CIITA. Accumulating evidences have shown that inhibition of EGFR through EGFR-TKIs can regulate the tumor immune microenvironment to achieve optimal anti-tumor activity of immunotherapy and improve therapeutic effects of PD-1/PD-L1 inhibitors (21). As a third-generation EGFR TKI, oxitinib has good blood-brain barrier permeability. Recently, Makhlin et al. successfully treated a patient with recurrent GBM by 2 mutation sites of EGFR C628F and A289V using oxitinib (22). The WES result of this patient suggested the presence of EGFR activating mutations, and so she underwent treatment with the combination of oxitinib. The patient tolerated the treatment well, and no treatment-related adverse reactions such as rash, diarrhea, mucositis, were observed. If the common adverse reactions exist, symptomatic treatment and reducing dose are needed according to specific situation.

This new treatment option in our GBM patient who was intolerant of chemotherapy was successful. However, the limitations of our study include the nature of work, only one case report, and lack of prognostic biomarkers. So, large cohort studies or clinical trials should be launched to verify the efficacy and safety of this strategy and find proper patients with potential biomarker benefiting from this strategy.

We have reported a GBM case with unmethylated MGMT promoter and intolerance of chemotherapy. After individualized immunization combined with anti-vascular and targeted therapy, there was no disease progression for nearly 11 months, significantly exceeding the historical data. At the same time, the most likely complications, such as allergic reaction, reactive capillary hyperplasia, rash, diarrhea and so on, didn't appear. So individualized combination therapy without chemotherapy may be suitable for such newly diagnosed GBM patients.

\section{Acknowledgments}

Funding: None.

\section{Footnote}

Reporting Checklist: The authors have completed the
CARE reporting checklist. Available at https://dx.doi. org/10.21037/atm-21-4625

Conflicts of Interest: All authors have completed the ICMJE uniform disclosure form (available at https://dx.doi. org/10.21037/atm-21-4625). The authors have no conflicts of interest to declare.

Ethical Statement: The authors are accountable for all aspects of the work in ensuring that questions related to the accuracy or integrity of any part of the work are appropriately investigated and resolved. All procedures performed in studies involving human participants were in accordance with the ethical standards of the institutional and/or national research committee(s) and with the Helsinki Declaration (as revised in 2013). Written informed consent was obtained from the patient's families for publication of this case report and accompanying images. A copy of the written consent is available for review by the editorial office of this journal.

Open Access Statement: This is an Open Access article distributed in accordance with the Creative Commons Attribution-NonCommercial-NoDerivs 4.0 International License (CC BY-NC-ND 4.0), which permits the noncommercial replication and distribution of the article with the strict proviso that no changes or edits are made and the original work is properly cited (including links to both the formal publication through the relevant DOI and the license). See: https://creativecommons.org/licenses/by-nc-nd/4.0/.

\section{References}

1. Fan X, Xiong Y, Wang Y. A reignited debate over the cell(s) of origin for glioblastoma and its clinical implications. Front Med 2019;13:531-9.

2. Stupp R, Hegi ME, Mason WP, et al. Effects of radiotherapy with concomitant and adjuvant temozolomide versus radiotherapy alone on survival in glioblastoma in a randomised phase III study: 5-year analysis of the EORTC-NCIC trial. Lancet Oncol 2009;10:459-66.

3. Brennan CW, Verhaak RG, McKenna A, et al. The somatic genomic landscape of glioblastoma. Cell 2013;155:462-77.

4. Gilbert MR, Wang M, Aldape KD, et al. Dose-dense temozolomide for newly diagnosed glioblastoma: a randomized phase III clinical trial. J Clin Oncol 2013;31:4085-91.

5. Iwai Y, Ishida M, Tanaka Y, et al. Involvement of PD-L1 
on tumor cells in the escape from host immune system and tumor immunotherapy by PD-L1 blockade. Proc Natl Acad Sci U S A 2002;99:12293-7.

6. Viteri S, Cabrera-Gálvez C, Rosell R. Keynote 407: the combination of pembrolizumab and chemotherapy cracks the shell of squamous cell lung cancer. Transl Lung Cancer Res 2020;9:828-32.

7. Omuro A, Vlahovic G, Lim M, et al. Nivolumab with or without ipilimumab in patients with recurrent glioblastoma: results from exploratory phase I cohorts of CheckMate 143. Neuro Oncol 2018;20:674-86.

8. Filley AC, Henriquez M, Dey M. Recurrent glioma clinical trial, CheckMate-143: the game is not over yet. Oncotarget 2017;8:91779-94.

9. Reardon DA, Brandes AA, Omuro A, et al. Effect of nivolumab vs bevacizumab in patients with recurrent glioblastoma: the CheckMate 143 phase 3 randomized clinical trial. JAMA Oncol 2020;6:1003-10.

10. Carrillo JA, Kesari S. Is re-radiation for glioblastoma after progression associated with increased survival: to treat or not to treat? Transl Cancer Res 2019;8:4-6.

11. Zeng J, See AP, Phallen J, et al. Anti-PD-1 blockade and stereotactic radiation produce long-term survival in mice with intracranial gliomas. Int J Radiat Oncol Biol Phys 2013;86:343-9.

12. Khan MI, Ostadhadi S, Mumtaz F, et al. Thalidomide attenuates the development and expression of antinociceptive tolerance to $\mu$-opioid agonist morphine through 1-arginine-iNOS and nitric oxide pathway. Biomed Pharmacother 2017;85:493-502.

13. Peters KB. Revisiting anti-angiogenic therapy for recurrent glioblastoma. Transl Cancer Res 2019;8:S569-72.

14. Bouzin C, Brouet A, De Vriese J, et al. Effects of vascular endothelial growth factor on the lymphocyte-endothelium interactions: identification of caveolin-1 and nitric oxide as control points of endothelial cell anergy. J Immunol 2007;178:1505-11.

15. Motz GT, Santoro SP, Wang LP, et al. Tumor endothelium FasL establishes a selective immune barrier promoting tolerance in tumors. Nat Med 2014;20:607-15.

16. Ohm JE, Gabrilovich DI, Sempowski GD, et al. VEGF inhibits T-cell development and may contribute to tumorinduced immune suppression. Blood 2003;101:4878-86.

17. Allen E, Jabouille A, Rivera LB, et al. Combined antiangiogenic and anti-PD-L1 therapy stimulates tumor immunity through HEV formation. Sci Transl Med 2017;9:eaak9679.

18. Manzoni M, Rovati B, Ronzoni M, et al. Immunological effects of bevacizumab-based treatment in metastatic colorectal cancer. Oncology 2010;79:187-96.

19. Duerinck J, Du Four S, Vandervorst F, et al. Randomized phase II study of axitinib versus physicians best alternative choice of therapy in patients with recurrent glioblastoma. J Neurooncol 2016;128:147-55.

20. Guo X, Wang S, Wang Y, et al. Anti-PD-1 plus antiVEGF therapy in multiple intracranial metastases of a hypermutated, IDH wild-type glioblastoma. Neuro Oncol 2021;23:699-701.

21. Dominguez C, Tsang KY, Palena C. Short-term EGFR blockade enhances immune-mediated cytotoxicity of EGFR mutant lung cancer cells: rationale for combination therapies. Cell Death Dis 2016;7:e2380.

22. Makhlin I, Salinas RD, Zhang D, et al. Clinical activity of the EGFR tyrosine kinase inhibitor osimertinib in EGFRmutant glioblastoma. CNS Oncol 2019;8:CNS43.

(English Language Editor: J. Jones)
Cite this article as: Wang Z, Du F, Ren Y, Jiang W. Treatment of MGMT promoter unmethylated glioblastoma with PD-1 inhibitor combined with anti-angiogenesis and epidermal growth factor receptor tyrosine kinase inhibitor: a case report. Ann Transl Med 2021;9(19):1508. doi: 10.21037/atm-21-4625 